$\underline{\text { Iranian Journal of Pathology }}$ | ISSN: 2345-3656

\title{
Alpha-Synuclein Expression in Acute Erythroleukaemia, Acute Megakaryoblastic Leukemia, and Normal Counterparts in Bone Marrow
}

Farid Kosari*, Sanam Akbarzadeh Hosseini, Hiva Saffar

Dept. of Pathology, Shariati Hospital, Tehran University of Medical Sciences, Tehran, Iran.

\begin{tabular}{l} 
KEYWORDS \\
\hline \\
Acute Myeloblastic Leukemia \\
AML-M6, AML-M7 \\
$\alpha$ - Synuclein
\end{tabular}

Article Info

Received 12 Oct 2015;

Accepted 11 Apr 2016;

Published Online 30 Aug 2016;

\section{ABSTRACT}

Background: Alpha-synuclein is a member of synuclein family of proteins with unidentified function localized in the cytoplasm, mitochondria of neurons, and presynaptic nerve endings. Although it is found in the Lewy bodies in synucleinopathies and in Alzheimer's disease, the protein could also be considered as a novel marker in diagnosis of diseases related to the hematopoietic system. Methods: The current study evaluated alpha-synuclein expression in bone marrow sections obtained from 9 patients with acute myeloblastic leukemia (AML)-M6, 2 patients with AML-M7, and 56 patients with other forms of AML by immunohistochemical (IHC) analysis

Results: Seven out of 9 cases with erythroleukemia (66.7\%) and 1 of the 2 cases with M7 (50\%) were positive. In contrast; the blasts in 2 out of 56 AML cases with non-M6/M7 (3.6\%) showed positive staining. Accordingly, alpha-synuclein was positive in normal erythroid precursors and megakaryocytes (if existing) in these cases; while, it was negative in lymphoid and myeloid precursors.

Conclusion: Alpha-synuclein expression in non-neoplastic and neoplastic erythroid cells and megakaryocytes could be used as a complementary and useful marker for distinction between AML-M6/M7 and other types of AML.

Corresponding Information: Dr Farid Kosari. Department of Pathology Department, Shariati Hospital, Kargar St., Tehran, Iran. Tel: 0098 9123435346. Email: faridkosari@yahoo.com

Copyright $\odot$ 2017, IRANIAN JOURNAL OF PATHOLOGY. This is an open-access article distributed under the terms of the Creative Commons Attribution-noncommercial 4.0 International License which permits copy and redistribute the material just in noncommercial usages, provided the original work is properly cited.

\section{Introduction}

Acute myeloblastic leukemia (AML) is a group of hematologic neoplasms with clinical similarity and different morphological, immunophenotypical, cytogenetic, and molecular features (1). Erythroleukaemia (AML-M6) and acute megakaryoblastic leukemia (AML-M7,) are considered as rare subtypes of AML with poor prognosis (2-6).

Alpha-synuclein is a protein, plentiful in the human brain, but in lower amount in heart and skeletal muscles (7-11).This protein belongs to a larger family of $\alpha, \beta$, and $\gamma$-synucleins, small (14-17 $\mathrm{kD})$ proteins with highly conserved amino acid termini and variable carboxy termini. The gene of each individual protein is designed in distinct chromosome (11-13).
Alpha-synuclein is the main part of the Lewy body, intracytoplasmic hyaline inclusion that is present in the nervous system in synucleinopathies such as Parkinson disease and multiple system atrophy.

Several studies showed that in the hematopoietic system, $\alpha$-synuclein is present in megakaryocytes and platelets (14), as well as erythroid precursors and erythrocytes (15-16).

Diagnosis of AML-M6 or AML-M7 is a serious challenge compared to other types of acute myeloid leukemia (20). Furthermore, in patients with AMLM6 and AML-M7, the blasts show some shared phenotypes informing that may arise from a common bipotent progenitor (21-23).

Currently, few data are available on incidence of $\alpha$-synuclein positive staining in AML-M6 and 
AML-M7. Considering the importance of obtaining accurate estimations about the incidence and stain intensity of $\alpha$-synuclein, the current study aimed at measuring the incidence of $\alpha$-synuclein positivity in the patients with AML.

\section{Materials and Methods}

Fifty The current observational study screened the data available in Central Hematopathology Lab in Shariati Hospital, Tehran, Iran, from 2009 to 2013. Sixty-seven cases with AML were selected; including 9 cases with AML-M6, 2 cases with AML-M7, and 56 cases with other forms of AML. The cases had been diagnosed based on the morphology of the bone marrow aspiration and biopsy and immunophenotyping of the blasts by immunohistochemical (IHC) assessment of the bone marrow biopsy and flow cytometry of the bone marrow aspirates. The paraffin blocks were retrieved from the Departments of Pathology at Shariati Hospital.

\section{Immunohistochemistry}

Bone marrow biopsy tissue sections were sliced in 3- $\mu \mathrm{m}$ thickness pieces. After baking at $65^{\circ} \mathrm{C}$ for 2 hours; slides were soaked in xylene for deparaffinization; then, $100 \%$ and $96 \%$ alcohols for rehydration and distilled water $(\mathrm{dH} 20)$ for washing. For antigen retrieval, $1.5 \mathrm{~mL}$ of $0.01 \mathrm{M}$ buffer citrate with $\mathrm{pH}=9$ was placed in autoclave $\left(121^{\circ} \mathrm{C}, 200\right.$ $\mathrm{kPa}, 25$ minutes); then, tissue sections were washed with distilled water $(\mathrm{dH} 20)$ and - soaked in pure formic acid for 1.5 minutes and washed again; then, the blocking antibody (normal goat serum) was applied and left for 20 minutes at room temperature. The slides were incubated with a 1:10 dilution of a mouse anti-human $\alpha$-synuclein monoclonal antibody for 2 hours. After washing with Trisbuffered saline (TBS), array slide was incubated with an appropriate secondary anti-mouse antibody reagent for 30 minutes and washed again with TBS. Applying 3,3'-Diaminobenzidine (DAB) substrate, the chromogen was proceeded in 10 minutes and final washing was done. The sections were stained with hematoxylin followed by exposure to bicarbonate for 30 seconds and finally dehydrated. Thereafter, samples were ready for mounting. Samples were evaluated separately by 2 pathologists.

Megakaryocytes were evaluated for the staining in low (X40) power field and erythroid cells in high (X400) power field.

Differences in intensity of $\alpha$-synuclein staining were scored as: $0=$ none, $1=$ weak, $2=$ average, and $3=$ strong staining.

Moreover, percentage of positive staining was evaluated in erythroid series, megakaryocytes, and blast cells, and scored from $0 \%$ up to $100 \%$.

\section{Statistical Analysis}

Data were analyzed by SPSS version 19.0 (Chicago, IL, USA) for windows, using Chi-square test with confidence interval of $95 \%$ and $\alpha=0.05$.

\section{Results}

\section{A: Percentage of positivity in the blasts}

The erythroblasts in 7 out of 9 cases with AMLM6 showed strong and diffuse cytoplasmic staining for $\alpha$-synuclein (Table 1), although myeloblasts were negative in all of the cases with M6 (Figure 3).

Table. 1 .Comparison of Staining of $\alpha$-Synuclein in Blasts in the Cases with AML

\begin{tabular}{cccc}
\hline & Total Patients, $\mathbf{n}$ & Positive Cases, $\mathbf{n}(\boldsymbol{\%})$ & Positivity in the Blast (\%) \\
\hline AML-M6 & 9 & $6^{*}(66.7)$ & $20 \%-100 \%$ \\
AML-M7 & 2 & $1^{\wedge}(50)$ & $100 \%$ \\
AML non M6/7 & 56 & $2(3.6)$ & $50 \%$ \\
\hline *Positive staining in erythroblasts & \multicolumn{2}{c}{ ^Positive staining in megakaryoblasts } & AML, acute myeloblastic leukemia
\end{tabular}

One of the 2 cases with AML-M7 showed intense positive cytoplasmic staining in the morphologically recognizable megakaryocyte and megakaryoblasts; however, myeloblasts were negative (Table 1) (Figure2). 


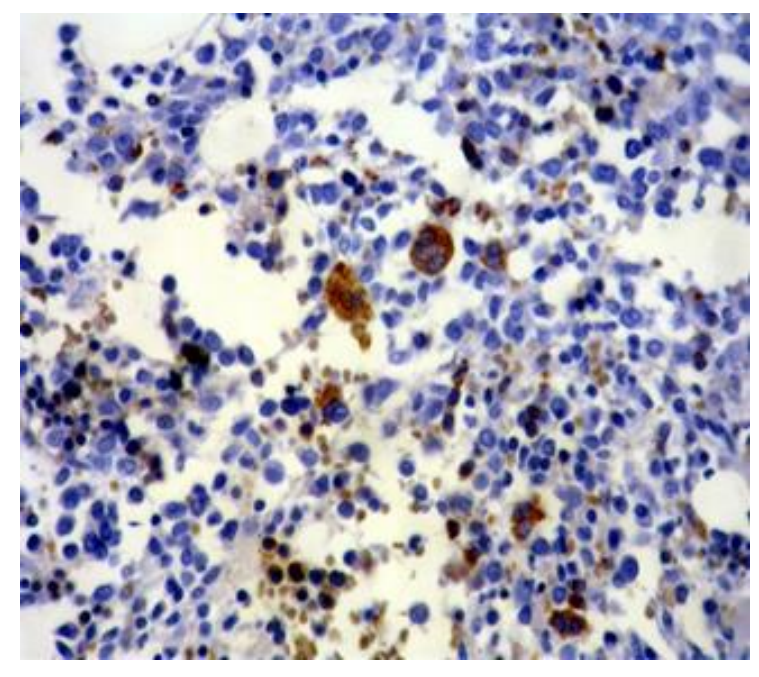

Fig 1. Normal bone marrow stained by $\alpha$ synuclein. Erythroid precursors, RBCs, and megakaryocytes show strong staining, while the other cells are negative (x200).

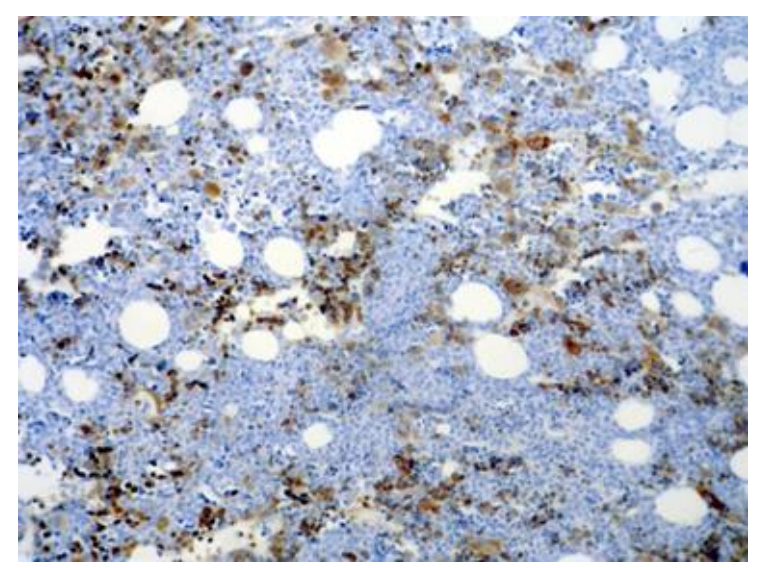

Fig 2. $\alpha$-synuclein staining in AML-M7; erythroid precursors and all of the abnormal megakaryocytes are positive, while myeloblasts in the background are negative (x100).

The incidence of positive staining for $\alpha$ synuclein in erythroblasts differs significantly in the AML- M6, compared to the non-AML M6/M7 group $(\mathrm{P}<0.001)$.

The incidence of positivity for $\alpha$-synuclein showed no significant difference between AML-M7 and non AML-M6/M7 groups ( $\mathrm{P}=0.102)$.

The incidence of positivity for $\alpha$-synuclein showed no significant difference between AML-M6 and AML-M7 $(\mathrm{P}=1.0)$.

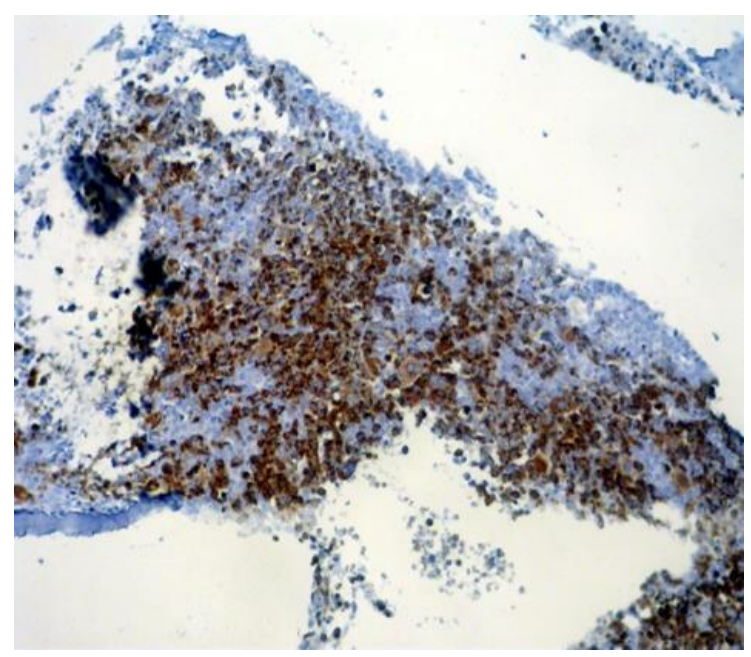

Fig 3. $\alpha$-synuclein staining in AML-M6; all of the erythroid precursors and few scattered megakaryocytes are positive, whereas non-crythroid blasts are negative (x100).

\section{B: Percentage of positivity in normal erythroid series and megakaryocytes:}

Of the 56 cases with non M6/M7, 39 cases had a significant number of normal erythroid lineage; 35 of which stained averagely $(2+)$ positive for $\alpha$ synuclein.

In 54 of the 56 cases, the non-neoplastic megakaryocyte were strongly (3+) positive for $\alpha$ synuclein. Erythroid precursors were positive for $\alpha$ synuclein in the cases with AML-M7 (Figure 2)

All of the megakaryocytes showed strong (3+) positive staining in cases with AML-M6 (Figure 3).

\section{Discussion}

This is the 2nd study to evaluate the efficacy of $\alpha$-synuclein antibody staining to detect erythroid and megakaryocytic lineages in hematopoietic malignancies. Recent data suggest that $\alpha$ - synuclein might be an important factor in acute myeloid leukemia (24). Among hematopoietic cells, $\alpha$ synuclein exists predominantly in megakaryocytes, platelets, erythroid precursors, and erythrocytes (1416). However, there is little knowledge about the distribution and presentation of this marker in normal and leukemic cells in different subtypes of AML. By applying new published $\alpha$-synuclein staining protocol, $\alpha$-synuclein expression can be evaluated in different types of AML (14-16, 24). 
In the current study, there was a significant difference in the positivity of $\alpha$-synuclein between M6 and non-M6/M7 cases. Although not all the cases in the study were positive as that of the Maitta et $\mathrm{al}^{24}$, both of them represent effectivity of this staining for distinction between the 2 groups.

Accordingly, in the current study only half of the cases with M7 showed positive $\alpha$-synuclein staining, which was in contrast with the results obtained by Maitta et al., showing positive staining in all 5 cases. This difference could be related to decreased number of M7 in the study and also to the quality of tissue processing.

The results of the current study showed that $\alpha$ synuclein was expressed as a lineage-related and cell-specific marker antigen in non-neoplastic erythroid and megakaryocyte lineage. The study found the incidence of average positive staining for this marker in non-neoplastic erythroid cells in $89.1 \%$ of the cases, and the incidence of positive staining in residual non neoplastic megakaryocytes was $94.7 \%$.

In the current study, staining intensity was more in mature megakaryocytes than in mature erythroid cells. In contrast to the present study, Maitta reported only minimal $\alpha$-synuclein staining in mature erythroid cells, both in normal reactive marrow and in neoplastic bone marrow disorders (24).

\section{Conclusion}

The current study concluded that $\alpha$-synuclein is expressed in erythroblasts, megakaryoblasts, mature erythroid cells, and megakaryocytes in normal bone marrow and in patients with AML. Expression of $\alpha$ synuclein in the blasts of acute erythroid leukemia and acute megakaryoblastic leukemia suggests that $\alpha$-synuclein could be a useful marker for the early detection of AML-M6, AML-M7, and the differential diagnosis from other types of AML. Therefore, $\alpha$-synuclein may be an applicable immunohistochemical marker in AML.

Conflict of Interest: The authors declare that there was no conflict of interest.

\section{References}

1. Maria R. Baer. Jhon P. Green et al. Acute Myeloid Leukemia in Adult; Wintrobe, s ClinicalHematology; 12th, 2009: p.79; 1883, 1849, 1852,1858 .

2. Colita A, Belhabri A, ChelghoumY, Charrin C, Fiere D, Thomas X. Prognosticfactors and treatment effects on survival in acute myeloid leukemia ofM6 subtype: A retrospective study of 54 cases. Annals of Oncology 2001; 12:451-455.

3. Wells AW, Bown N, Reid MM, Hamilton PJ, Jackson GH, Taylor PR. Erythroleukaemia in the north of England: a population based study.Journal of Clinical Pathology 2001: 54:608-612.

4. Domingo-Claros A, Larriba I, Rozman M, Irriguible D, Vallespi T, Aventin A,Ayats R, Milla F, Sole F, Florensa L, et al: Acute erythroid neoplasticproliferations. A biological study based on 62 patients. Haematologica2002: 87(2):148-153.

5. Rogelio Paredes-Aguilera LR-GNL-SRAT: Biology, clinical, and hematologicfeatures of acute megakaryoblastic leukemia in children. American Journal of Hematology 2003: 73:71-80.

6. Garderet L, Labopin M, Gorin NC, Polge E, Baruchel A, Meloni G, Ortega J,Vossen J, Bunjes D, Leverger G, et al: Hematopoietic stem celltransplantation for de novo acute megakaryocytic leukemia in firstcomplete remission: a retrospective study of the European Group forBlood and Marrow Transplantation (EBMT). Blood 2005: 105(1):405409.

7. George JM. The synucleins. Genome Biol 2002;3:Reviews 3002.

8. Nakai M, Fujita M, Waragai $M$, et al. Expression of alpha-synuclein, a presynapticprotein implicated in Parkinson's disease, in erythropoietic lineage. BiochemBiophys Res Commun 2007;358:104-110.

9. Barbour R, Kling K, Anderson JP, et al. Red blood cells are the major sourceof alpha-synuclein in blood. Neurodegener Dis 2008;5:55-59. 
10. Fung KM, Rorke LB, Giasson B, et al. Expression of alpha-, beta-, andgamma-synuclein in glial tumors and medulloblastomas. ActaNeuropathol2003;106:167-175.

11. Chen X, de Silva HA, Pettenati MJ, et al. The human NACP/alpha-synucleingene: Chromosome assignment to 4q21.3-q22 and TaqI RFLP analysis.Genomics 1995;26:425-427.

12. Spillantini MG, Divane A, Goedert $M$. Assignment of human alpha-synuclein(SNCA) and beta-synuclein (SNCB) genes to chromosomes 4q21 and 5q35.Genomics 1995;27:379-381.

13. Ninkina NN, Alimova-Kost MV, Paterson JW, et al. Organization, expression andpolymorphism of the human persyn gene. Hum Mol Genet 1998;7:1417-1424.

14. Hashimoto M, Yoshimoto M, Sisk A, et al. NACP, a synaptic protein involvedin Alzheimer's disease, is differentially regulated during megakaryocyte differentiation.BiochemBiophys Res Commun 1997;237:611-616.

15. Nakai M, Fujita M, Waragai M, et al. Expression of alpha-synuclein, a presynapticprotein implicated in Parkinson's disease, in erythropoietic lineage. BiochemBiophys Res Commun 2007;358:104-110.

16. Barbour R, Kling K, Anderson JP, et al. Red blood cells are the major sourceof alpha-synuclein in blood. Neurodegener Dis 2008; 5:55-59.

17. Ueda K, Fukushima H, Masliah E, et al. Molecular cloning of cDNA encodingan unrecognized component of amyloid in Alzheimer disease. Proc Natl AcadSci USA 1993;90: 1128211286
18. Goedert M. Alpha-synuclein and neurodegenerative diseases. Nat Rev Neurosci2001;2:492-501.

19. Takao M, Ghetti B, Yoshida H, et al. Earlyonset dementia with Lewy bodies.Brain Pathol 2004;14:137-147.

20. Park S, Picard F, Dreyfus F: Erythroleukemia. a need for a new definition.Leukemia 2002, 16(8):1399-1401.

21. Linari S, Vannucchi AM, Ciolli S, Leoni F, Caporale R, Grossi A, Pagliai G, Santini V, Paoletti F, Ferrini PR: Coexpression of erythroid and megakaryocytic genes in acute erythroblastic (FAB M6) and megakaryoblastic (FAB M7) leukaemias. British journal of haematology 1998; 102(5):13351337.

22. Debili N, Coulombel L, Croisille L, Katz A, Guichard J, Breton-Gorius J,Vainchenker W: Characterization of a bipotenterythromegakaryocyticprogenitor in human bone marrow. Blood 1996, 88(4):1284-1296.

23. Nakorn TN, Miyamoto T, Weissman IL: Characterization of mouseclonogenic megakaryocyte progenitors. Proceedings of the NationalAcademy of Sciences of the United States of America 2003, 100(1):205-210.

24. Maitta RW, Wolgast LR, Wang Q, Zhang H, Bhattacharyya P, Gong JZ, Sunkara J,Albanese JM, Pizzolo JG, Cannizzaro LA, Ramesh KH, Ratech H. Alpha- andbeta-synucleins are new diagnostic tools for acute erythroid leukemia and acutemegakaryoblastic leukemia. Am J Hematol. 2011; 86(2):230-4.

\section{How to Cite This Article:}

Kosari F, Akbarzadeh S, Saffar H. Alpha-Synuclein Expression in Acute Erythroleukaemia, Acute Megakaryoblastic Leukemia, and Normal Counterparts in Bone Marrow. Iranian Journal of Pathology. 2017;12(1):74-78. 